J Org Chem. 2008 October 3; 73(19): 7560-7564. doi:10.1021/jo8010683.

\title{
Synthesis of (-)-Hamigeran B
}

\author{
Douglass F. Taber and Weiwei Tian \\ Department of Chemistry and Biochemistry, University of Delaware, Newark, DE, 19716
}

\begin{abstract}
The synthesis of (-)-hamigeran B has been achieved, based on a new approach to cyclopentane construction, the rhodium-mediated intramolecular C-H insertion of $\alpha$-aryl- $\alpha$-diazo ketones. The endo isopropyl group was installed by selective hydrogenation of a cyclopropylidene substituent.
\end{abstract}

\section{Introduction}

(-)-Hamigeran B 1 is one of a family of eight hamigerans (Fig. 1) isolated ${ }^{1}$ in 2000 from the poecilosclerid sponge Hamigera tarangaensis, collected from the Hen and Chicken Islands east of New Zealand. Hamigeran B was the only one of the family to show antiviral activity, effecting complete inhibition of both herpes and polio viruses at low concentration and with only slight cytotoxicity.

A central concern in the total synthesis of the hamigerans is the construction of the cyclic quaternary center, from which the other two stereocenters may evolve. Three independent approaches to this problem have been described. Nicolaou ${ }^{2}$ first reported an asymmetric synthesis utilizing a [4+2] photocycloaddition. This route started from an enantiomericallypure epoxide, obtained via the Jacobsen hydrolytic kinetic resolution. Clive ${ }^{3}$ described a synthesis in which the chiral quaternary center was constructed using Meyers' chiral auxiliary. Trost ${ }^{4}$ installed the quaternary stereogenic center by Pd-catalyzed asymmetric allylic alkylation of a preformed cyclopentanone.

We envisioned that the key intermediate 12 (Scheme 1) could be assembled by convergent coupling of 9 with the enantiomerically-pure citronellol derivative 10. ${ }^{5,6} \mathrm{Rh}$-mediated intramolecular $\mathrm{C}-\mathrm{H}$ insertion, ${ }^{7}$ proceeding with retention of absolute configuration, ${ }^{8}$ would then complete the cyclopentane assembly while at the same time securing the enantiomericallypure quaternary center of $\mathbf{1 2}$. Our enthusiasm for this approach was, however, tempered by the earlier report ${ }^{9}$ that the $\alpha$-aryl- $\alpha$-diazoketone $\mathbf{1 3}$ could not be induced to cyclize to $\mathbf{1 4} .^{10}$

\section{Results and Discussion}

\section{Construction and cyclization of $\alpha$-aryl- $\alpha$-diazoketones}

In order to develop this approach to cyclopropane construction, two challenges (Scheme 2) had to be overcome. ${ }^{11}$ The first challenge was to optimize the base, solvent and sulfonyl azide for the diazo transfer reaction on an $\alpha$-aryl ketone. Overall, we found that 2,4,6triisopropylbenzenesulfonyl azide (TIBSA) was better than benzenesulfonyl azide, methanesulfonyl azide, 4-acetamidobenzenesulfonyl azide or p-nitrobenzenesulfonyl azide, especially on substrates with an electron-rich aromatic ring. As the solvent, toluene was better than dichloromethane or acetonitrile. In combination with DBU, TIBSA gave consistently high yields of $\alpha$-aryl- $\alpha$-diazo ketones. 
The next challenge was the Rh-mediated intramolecular C-H insertion. Because of the electrondonating aromatic substituent, dimer formation was always the main side reaction competing with $\mathrm{C}-\mathrm{H}$ insertion. By altering the temperature, the solvent, the addition sequence and the rhodium catalyst, the yields of the $\mathrm{C}-\mathrm{H}$ insertion could be improved. Under the optimized conditions (toluene as the solvent, rapid addition of the diazoketone into rhodium catalyst $\left.\mathrm{Rh}_{2}(\mathrm{pttl})_{4}\right){ }^{12}$ at room temperature), the diazo ketone $\mathbf{1 6}$ cyclized efficiently. We were pleased to observe that under these conditions, even 13 could be induced to cyclize to $\mathbf{1 4}$, albeit in modest yield.

\section{Synthesis of the tricyclic ketone 21}

The known aldehyde $\mathbf{1 0}^{6}$ (Scheme 3 ) was derived from $(R$ )-citronellol in two steps. Direct benzylic deprotonation of 3,5-dimethylanisole 9 by one equivalent of "super-base" 13 in THF followed by addition of the aldehyde $\mathbf{1 0}$ at low temperature led to the alcohol $\mathbf{1 8}$ as a mixture of two diastereomers. Phenethyl alcohols such as $\mathbf{1 8}$ are sometimes difficult to oxidize, and several reagents were ineffective. Fortunately, the PCC catalyzed oxidation with periodic acid $^{14}$ worked well.

With the ketone 19 in hand, we could bring to bear our earlier experience developing the cyclization protocol. Diazo transfer reaction by TIBSA and DBU in toluene was high yielding, delivering 11. The diazo ketone $\mathbf{1 1}$ was unusually unstable and light sensitive. A quick chromatography of $\mathbf{1 1}$ and immediate processing were required.

We were pleased to find, based on our previous optimization, that Rh-mediated $\mathrm{C}-\mathrm{H}$ insertion to form the cyclopentane also proceeded efficiently. The product was a mixture of two diastereomers at the $\alpha$ position, as expected. The two diastereomers could be separated by column chromatography, but interconverted on storage, so we carried the mixture on.

Removal of the benzyl protecting group by hydrogenation followed by oxidation with the DessMartin reagent gave the aldehyde 21, still as a mixture of $\alpha$-diastereomers. Under acid catalysis, the two diastereomers of the aldehyde $\mathbf{2 1}$ readily interconverted. The cis isomer of $\mathbf{2 1}$ could easily participate in the intramolecular Friedel-Crafts reaction, to form the cyclized benzylic alcohol. In situ, the alcohol underwent dehydration to give the alkene $\mathbf{2 2 . 1 5}$

\section{Synthesis of (-)-Hamigeran B}

Wittig reagents did not convert ketone $\mathbf{2 2}$ into the corresponding alkene, perhaps because $\mathbf{2 2}$ was quite acidic. The Petasis reagent, ${ }^{16}$ known to be nonbasic, was successful, delivering alkene 23 in $60 \%$ yield (Scheme 4 ). The strained alkene was unstable under the reaction condition $\left(55^{\circ} \mathrm{C}\right)$, easily isomerizing to the endocyclic cyclopentene during the reaction. We found that inclusion of $\mathrm{NaHCO}_{3}$ in the reaction mixture prevented the isomerization.

With the diene $\mathbf{2 3}$ in hand, we were again faced with two challenges, differentiating the two alkenes, and controlling the diastereoselectivity of the hydrogenation of the cyclopropylidene. The propensity of the alkene to move into and around the ring was exacerbated by hydrogenation catalysts. Ir black was finally tried because it was known to minimize such alkene migrations. ${ }^{4,17}$ The reduction was successful, delivering essentially a single diastereomer. We were pleased to observe that the strained cyclopropylidene could be hydrogenated without concomitant reduction of the benzylic alkene.

Upjohn dihydroxylation 3,18 of the alkene 24 led to the diol 25 as a mixture of diastereomers. Hydrogenolytic cleavage ${ }^{7 b, 19}$ of the cyclopropane ring at $50{ }^{\circ} \mathrm{C}$ by $\mathrm{PtO}_{2} / \mathrm{AcOH}$ established the isopropyl group and also removed the benzylic alcohol. Fortunately, oxidation of $\mathbf{2 6}$ with TBAP/NMO ${ }^{20}$ led directly to the diketone 27 , presumably by the oxidation of the enol form of the initially generated ketone. Demethylation and bromination following the literature 
precedent ${ }^{3,4}$ then led to (-)-hamigeran B 1, identical $\left({ }^{1} \mathrm{H}\right.$ NMR, $\left.{ }^{13} \mathrm{C} N M R,[\alpha]_{\mathrm{D}}\right)$ with natural and previously synthesized material. ${ }^{1-4}$

\section{Conclusion}

We have developed a general route to the 6, 6, 5-tricyclic skeleton of (-)-hamigeran B based on $\mathrm{C}-\mathrm{H}$ insertion of an $\alpha$-aryl- $\alpha$-diazoketone followed by Friedel-Crafts cyclization. The installation of the endo isopropyl group was achieved by selective hydrogenation of a cyclopropylidene substituent. We expect that $\mathrm{Rh}$-mediated intramolecular $\mathrm{C}-\mathrm{H}$ insertion of $\alpha$ aryl- $\alpha$-diazo ketones will be a powerful tool for the synthesis of natural products of biological interest.

\section{Experimental Section}

\section{(5R)-7-(Benzyloxy)-1-(3-methoxy-5-methylphenyl)-5-methyl-1-diazoheptan-2-one (11)}

To $300 \mathrm{~mL}$ of toluene were added the ketone $19(10.0 \mathrm{~g}, 28.2 \mathrm{mmol})$, DBU ( $8.6 \mathrm{~g}, 56.5 \mathrm{mmol})$ and 2,4,6-triisopropylbenzenesulfonylazide $(10.5 \mathrm{~g}, 33.9 \mathrm{mmol})$ sequentially at $0{ }^{\circ} \mathrm{C} .{ }^{11}$ The reaction mixture was maintained in darkness and stirred at $\mathrm{rt}$ for $4 \mathrm{~h}$. The reaction mixture was directly chromatographed to afford the diazo ketone $\mathbf{1 1}$ as a yellow oil $(9.66 \mathrm{~g}, 25.4 \mathrm{mmol}$, $90 \%$ yield $)$. TLC $R_{f}(\mathrm{PE} / \mathrm{MTBE}=8 / 2)=0.50 ;[\alpha]^{20} \mathrm{D}=+3.93^{\circ}\left(\mathrm{c} 2.80, \mathrm{CH}_{2} \mathrm{Cl}_{2}\right) ;{ }^{1} \mathrm{H}$ NMR $\delta$ $0.90(3 \mathrm{H}, \mathrm{d}, \mathrm{J}=6.8 \mathrm{~Hz}), 1.60(5 \mathrm{H}, \mathrm{m}), 2.31(3 \mathrm{H}, \mathrm{s}), 2.55(2 \mathrm{H}, \mathrm{m}), 3.49(2 \mathrm{H}, \mathrm{m}), 3.77(3 \mathrm{H}, \mathrm{s})$, $4.47(2 \mathrm{H}, \mathrm{s}), 6.60(1 \mathrm{H}, \mathrm{s}), 6.82(1 \mathrm{H}, \mathrm{s}), 6.97(1 \mathrm{H}, \mathrm{s}), 7.27(5 \mathrm{H}, \mathrm{m}) ;{ }^{13} \mathrm{C} \mathrm{NMR} \delta \mathrm{u}^{21} 31.6,36.5$, 36.9, 68.4, 72.1, 73.0, 126.6, 138.6, 140.0, 160.1, 193.0; d 19.4, 21.7, 29.7, 55.2, 108.6, 113.6, 118.6, 127.5, 127.6, 128.3; IR (film, cm ${ }^{-1}$ ) 2927, 2073, 1649, 1592, 1240; HRMS calcd for $\mathrm{C}_{23} \mathrm{H}_{28} \mathrm{O}_{3}\left(\mathrm{M}-\mathrm{N}_{2}\right)$ 352.2038, obsd 352.2041.

\section{(3S)-3-(2-(Benzyloxy)ethyl)-2-(3-methoxy-5-methylphenyl)-3-methylcyclopentanone (12)}

$\mathrm{Rh}_{2}(\mathrm{R}-\mathrm{pttl})_{4}{ }^{12}(5 \mathrm{mg})$ was dissolved in $2 \mathrm{~mL}$ of toluene. To this solution was added a solution of the diazoketone $11(140 \mathrm{mg}, 0.368 \mathrm{mmol})$ in $2.0 \mathrm{~mL}$ of toluene at rt within $1 \mathrm{~min}$. The reaction was continued for an additional $15 \mathrm{~min}$ at $\mathrm{rt}$. Then the reaction mixture was concentrated and chromatographed to afford the ketone $\mathbf{1 2}$ as a mixture of two diastereomers (108 mg, $0.305 \mathrm{mmol}, 83 \%$ yield). TLC $R_{f}(\mathrm{PE} / \mathrm{MTBE}=8 / 2)=0.22 ;{ }^{1} \mathrm{H}$ NMR $\delta 0.79(1.2 \mathrm{H}$, s), $1.18(1.8 \mathrm{H}, \mathrm{s}), 1.50(1.0 \mathrm{H}, \mathrm{m}), 1.67(1.0 \mathrm{H}, \mathrm{m}), 1.83(1.0 \mathrm{H}, \mathrm{m}), 1.94(1.0 \mathrm{H}, \mathrm{m}), 2.31(1.0 \mathrm{H}$, $\mathrm{m}), 2.34(3.0 \mathrm{H}, \mathrm{s}), 2.42(1.0 \mathrm{H}, \mathrm{m}), 3.11(0.6 \mathrm{H}, \mathrm{s}), 3.24(0.4 \mathrm{H}, \mathrm{s}), 3.4-3.6(2.0 \mathrm{H}, \mathrm{m}), 3.74(1.2 \mathrm{H}$, s), $3.75(1.8 \mathrm{H}, \mathrm{s}), 4.41(1.2 \mathrm{H}, \mathrm{s}), 4.49(0.8 \mathrm{H}, \mathrm{s}), 6.43(2.0 \mathrm{H}, \mathrm{m}), 6.62(1.0 \mathrm{H}, \mathrm{s}), 7.28(5 \mathrm{H}$, $\mathrm{m}) ;{ }^{13} \mathrm{C}$ NMR $\delta$ u $32.3,33.2,33.8,35.9,36.1,40.8,43.2,43.3,67.0,67.2,73.2,73.3,135.9$, 136.2, 138.3, 139.0, 139.1, 159.3, 159.4, 217.6, 217.9; d 20.7, 21.7, 21.8, 26.1, 55.2, 67.4, 68.9, 113.2, 113.3, 113.4, 113.6, 123.5, 123.9, 127.7, 128.5; IR (film, $\mathrm{cm}^{-1}$ ) 2959, 1724, 1173, 1068; HRMS calcd for $\mathrm{C}_{23} \mathrm{H}_{28} \mathrm{O}_{3}\left(\mathrm{M}^{+}\right)$352.2038, obsd 352.2023.

(3S)-3-(2-Hydroxyethyl)-2-(3-methoxy-5-methylphenyl)-3-methylcyclopentanone (20)

The ketone $12(6.03 \mathrm{~g}, 17.1 \mathrm{mmol})$ was dissolved in $50 \mathrm{~mL}$ of THF and $1 \mathrm{~mL}$ of water. $5 \%$ $\mathrm{Pd} / \mathrm{C}(1.0 \mathrm{~g})$ was added at rt. The reaction container was then filled with hydrogen gas ( $1 \mathrm{~atm})$ and stirred vigorously overnight. The mixture was passed through a pad of celite, then dried $\left(\mathrm{Na}_{2} \mathrm{SO}_{4}\right)$ and concentrated in vacuo. The residue was chromatographed to afford the alcohol 20 as a mixture of two diastereomers $(4.41 \mathrm{~g}, 16.8 \mathrm{mmol}, 98 \%$ yield $)$. TLC $R_{f}(\mathrm{PE} / \mathrm{MTBE}=$ $6 / 4)=0.10 ;{ }^{1} \mathrm{H}$ NMR $\delta 0.80(1.8 \mathrm{H}, \mathrm{s}), 1.19(1.2 \mathrm{H}, \mathrm{s}), 1.2-1.4(1 \mathrm{H}, \mathrm{m}), 1.7-1.9(3 \mathrm{H}, \mathrm{m}), 2.17$ $(1 \mathrm{H}, \mathrm{m}), 2.30(3 \mathrm{H}, \mathrm{s}), 2.47(1 \mathrm{H}, \mathrm{m}), 3.11(0.4 \mathrm{H}, \mathrm{s}), 3.20(0.6 \mathrm{H}, \mathrm{s}), 3.6(1 \mathrm{H}, \mathrm{m}), 3.7(2 \mathrm{H}, \mathrm{m})$, $3.75(3 \mathrm{H}, \mathrm{s}), 6.42(2 \mathrm{H}, \mathrm{m}), 6.63(1 \mathrm{H}, \mathrm{s}) ;{ }^{13} \mathrm{C}$ NMR $\delta$ u $32.1,33.3,35.8,35.9,36.8,43.1,43.2$, 43.7, 59.2, 59.4, 135.9, 136.1, 139.0, 139.1, 159.3, 159.4, 217.6, 217.9; d 20.4, 21.7, 26.0, 55.2, 67.5, 68.8, 113.2, 113.2, 113.4, 113.6, 123.4, 123.8; IR (film, $\mathrm{cm}^{-1}$ ) 3428, 2952, 1733, 1595, 1459; HRMS calcd for $\mathrm{C}_{16} \mathrm{H}_{22} \mathrm{O}_{3}\left(\mathrm{M}^{+}\right) 262.1569$, obsd 262.1557. 
(1S)-2-(2-(3-Methoxy-5-methylphenyl)-1-methyl-3-oxocyclopentyl)acetaldehyde (21)

Dess-Martin periodinane $(7.42 \mathrm{~g}, 17.5 \mathrm{mmol})$ was added into the solution of the alcohol 20 (3.06 g, $11.7 \mathrm{mmol})$ in $100 \mathrm{~mL}$ of DCM at rt. The reaction continued for $30 \mathrm{~min}$. The mixture was diluted with $300 \mathrm{~mL}$ of ether and passed through a pad of silica gel. The eluant was concentrated in vасио. The residue was chromatographed to afford the ketone $\mathbf{2 1}$ as a mixture of two diastereomers $(2.90 \mathrm{~g}, 11.1 \mathrm{mmol}, 95 \%$ yield $)$. TLC $R_{f}(\mathrm{PE} / \mathrm{MTBE}=6 / 4)=0.29 ;{ }^{1} \mathrm{H}$ NMR $\delta 0.94(0.6 \mathrm{H}, \mathrm{s}), 1.40(2.4 \mathrm{H}, \mathrm{s}), 1.90(1 \mathrm{H}, \mathrm{m}), 2.14(2 \mathrm{H}, \mathrm{m}), 2.23(1.0 \mathrm{H}, \mathrm{m}), 2.32(3 \mathrm{H}$, s), $2.50(2 \mathrm{H}, \mathrm{m}), 3.19(0.8 \mathrm{H}, \mathrm{s}), 3.41(0.2 \mathrm{H}, \mathrm{s}), 3.77(3 \mathrm{H}, \mathrm{s}), 6.39(1 \mathrm{H}, \mathrm{s}), 6.43(1 \mathrm{H}, \mathrm{s}), 6.65$ $(1 \mathrm{H}, \mathrm{s}), 9.59(0.8 \mathrm{H}, \mathrm{t}, \mathrm{J}=2.4 \mathrm{~Hz}), 9.83(0.2 \mathrm{H}, \mathrm{t}, \mathrm{J}=2.4 \mathrm{~Hz}) ;{ }^{13} \mathrm{C}$ NMR $\delta$ u 33.0, 33.1, 35.8, 36.2, 43.3, 43.7, 48.9, 54.4, 135.6, 135.9, 139.7, 140.1, 159.9, 160.1, 216.4, 217.4; d 21.6, 22.1, 26.7, 55.6, 66.7, 68.3, 113.7, 113.9, 114.0, 123.5, 124.0, 202.3, 202.4; IR (film, $\mathrm{cm}^{-1}$ ) 2958, 2739, 1735, 1597, 1287; HRMS calcd for $\mathrm{C}_{16} \mathrm{H}_{20} \mathrm{O}_{3}\left(\mathrm{M}^{+}\right)$260.1412, obsd 260.1408.

\section{(3aS,9bS)-6-Methoxy-3a,8-dimethyl-3,3a-dihydro-2H-cyclopenta[ $\alpha$ ]naphthalen-1(9ßH)-one} (22)

The aldehyde 21 ( $0.407 \mathrm{~g}, 1.56 \mathrm{mmol})$ was dissolved in $15 \mathrm{~mL}$ of diethyl ether at $\mathrm{rt}$. $\mathrm{BF}_{3} \cdot \mathrm{OEt}_{2}(5 \mathrm{~mL})$ was added at once. The reaction mixture turned blue right after the addition. The mixture was stirred for $1 \mathrm{~h}$ before quenching by water. The mixture was partitioned between MTBE and, sequentially, water, saturated aqueous $\mathrm{NaHCO}_{3}$ and brine. The combined organic extract was dried $\left(\mathrm{Na}_{2} \mathrm{SO}_{4}\right)$ and concentrated in vacuo. The residue was chromatographed to afford the alkene $\mathbf{2 2}$ as a colorless oil ( $0.138 \mathrm{~g}, 0.570 \mathrm{mmol}, 37 \%$ yield). $\operatorname{TLC} R_{f}(\mathrm{PE} / \mathrm{MTBE}=8 / 2)=0.33 ;[\alpha]^{20} \mathrm{D}=-109.8^{\circ}\left(\mathrm{c} 1.90, \mathrm{CH}_{2} \mathrm{Cl}_{2}\right) ;{ }^{1} \mathrm{H}$ NMR $\delta 1.12(3 \mathrm{H}, \mathrm{s})$, $1.81(1 \mathrm{H}, \mathrm{m}), 2.02(2 \mathrm{H}, \mathrm{m}), 2.29(3 \mathrm{H}, \mathrm{s}), 2.47(1 \mathrm{H}, \mathrm{m}), 2.83(1 \mathrm{H}, \mathrm{s}), 3.72(3 \mathrm{H}, \mathrm{s}), 5.48(1 \mathrm{H}$, $\mathrm{dd}, \mathrm{J}=1.2,9.6 \mathrm{~Hz}), 6.52(1 \mathrm{H}, \mathrm{s}), 6.57(1 \mathrm{H}, \mathrm{s}), 6.70(1 \mathrm{H}, \mathrm{d}, \mathrm{J}=10 \mathrm{~Hz}) ;{ }^{13} \mathrm{C}$ NMR $\delta$ u 35.5, $38.8,41.8,117.4,131.1,138.3,154.9,217.6$; d 21.9, 25.7, 55.5, 60.1, 110.8, 121.8, 123.5, 132.4; IR (film, $\mathrm{cm}^{-1}$ ) 2951, 1742, 1460, 1270, 1104; HRMS calcd for $\mathrm{C}_{16} \mathrm{H}_{18} \mathrm{O}_{2}\left(\mathrm{M}^{+}\right)$ 242.1307, obsd 242.1296.

\section{(3aS,9bR)-1-Cyclopropylidene-6-methoxy-3a,8-dimethyl-2,3,3a,9b-tetrahydro-1 H- cyclopenta[a]naphthalene (23)}

The ketone $22(0.185 \mathrm{~g}, 0.763 \mathrm{mmol})$ and $\mathrm{NaHCO}_{3}(12 \mathrm{mg})$ were added to $5 \mathrm{~mL}$ of toluene. Petasis reagent ${ }^{16}(6.5 \mathrm{~mL}, 0.7 \mathrm{M}$ in toluene, $4.58 \mathrm{mmol})$ was added at $\mathrm{rt}$. The reaction mixture was maintained at $55-60^{\circ} \mathrm{C}$ for 2 days, then chromatographed directly. The alkene $\mathbf{2 3}$ was isolated as yellow oil $(0.120 \mathrm{~g}, 0.45 \mathrm{mmol}, 60 \%$ yield $)$. TLC $R_{f}(\mathrm{PE} / \mathrm{MTBE}=8 / 2)=0.80$; $[\alpha]^{20}{ }_{\mathrm{D}}=+103.8^{\circ}\left(\mathrm{c} 6.26, \mathrm{CH}_{2} \mathrm{Cl}_{2}\right) ;{ }^{1} \mathrm{H}$ NMR $\delta 0.49(1 \mathrm{H}, \mathrm{m}), 0.69(1 \mathrm{H}, \mathrm{m}), 0.76(1 \mathrm{H}, \mathrm{m}), 0.87$ $(1 \mathrm{H}, \mathrm{m}), 1.10(3 \mathrm{H}, \mathrm{s}), 1.75(1 \mathrm{H}, \mathrm{m}), 1.90(1 \mathrm{H}, \mathrm{m}), 2.30(1 \mathrm{H}, \mathrm{m}), 2.33(3 \mathrm{H}, \mathrm{s}), 2.50(1 \mathrm{H}, \mathrm{m})$, $3.29(1 \mathrm{H}, \mathrm{bs}), 3.78(3 \mathrm{H}, \mathrm{s}), 5.51(1 \mathrm{H}, \mathrm{d}, \mathrm{J}=9.6 \mathrm{~Hz}), 6.52(1 \mathrm{H}, \mathrm{s}), 6.69(1 \mathrm{H}, \mathrm{s}), 6.70(1 \mathrm{H}, \mathrm{d}, \mathrm{J}$ $=10 \mathrm{~Hz}) ;{ }^{13} \mathrm{C}$ NMR $\delta$ u $1.0,2.7,32.4,39.8,44.1,113.0,117.4,136.6,136.7,154.6 ; \mathrm{d} 21.8$, 24.8, 54.1, 55.3, 109.3, 119.9, 124.0, 134.4; IR (film, $\left.\mathrm{cm}^{-1}\right)$ 2919, 2837, 1571, 1459, 1324, 1095; HRMS calcd for $\mathrm{C}_{19} \mathrm{H}_{22} \mathrm{O}\left(\mathrm{M}^{+}\right)$266.1671, obsd 266.1660 .

\section{(1R,3aS,9bS)-1-Cyclopropyl-6-methoxy-3a,8-dimethyl-2,3,3a,9b-tetrahydro-1H-cyclopenta [a]naphthalene (24)}

The alkene $23(0.36 \mathrm{~g}, 1.35 \mathrm{mmol})$ and $\mathrm{Ir} \mathrm{black}^{4}(50 \mathrm{mg})$ were mixed with $10 \mathrm{~mL}$ of EtOH at rt. The mixture was then placed in a Parr reactor filled with $\mathrm{H}_{2}(1100 \mathrm{psi})$. The reaction was monitored by GC-MS. After about 4-8 h, the reaction mixture was diluted with diethyl ether $(100 \mathrm{~mL})$ and passed through a pad of celite. The eluant was concentrated and chromatographed to afford 24 as a colorless oil $(0.201 \mathrm{~g}, 0.749 \mathrm{mmol}, 55 \%$ yield, $80 \%$ yield based on starting material not recovered). Starting material was recovered $\left(0.11 \mathrm{~g}, 31 \%\right.$ yield, TLC $R_{f}(\mathrm{PE} / \mathrm{DCM}$ $=8.5 / 1.5)=0.29)$. TLC $R_{f}(\mathrm{PE} / \mathrm{DCM}=8.5 / 1.5)=0.43 ;[\alpha]^{20} \mathrm{D}=+10.1^{\circ}\left(\mathrm{c} 6.22, \mathrm{CH}_{2} \mathrm{Cl}_{2}\right) ;{ }^{1} \mathrm{H}$ NMR $\delta-0.39(1 \mathrm{H}, \mathrm{m}),-0.21(1 \mathrm{H}, \mathrm{m}),-0.14(1 \mathrm{H}, \mathrm{m}), 0.06(1 \mathrm{H}, \mathrm{m}), 0.23(1 \mathrm{H}, \mathrm{m}), 1.04(3 \mathrm{H}, \mathrm{s})$, 
$1.36(1 \mathrm{H}, \mathrm{m}), 1.49(1 \mathrm{H}, \mathrm{m}), 1.58(1 \mathrm{H}, \mathrm{m}), 1.85(2 \mathrm{H}, \mathrm{m}), 2.31(3 \mathrm{H}, \mathrm{s}), 2.85(1 \mathrm{H}, \mathrm{d}, \mathrm{J}=10 \mathrm{~Hz})$, $3.80(3 \mathrm{H}, \mathrm{s}), 5.56(1 \mathrm{H}, \mathrm{d}, \mathrm{J}=10 \mathrm{~Hz}), 6.50(1 \mathrm{H}, \mathrm{s}), 6.54(1 \mathrm{H}, \mathrm{s}), 6.66(1 \mathrm{H}, \mathrm{d}, \mathrm{J}=10 \mathrm{~Hz}) ;{ }^{13} \mathrm{C}$ NMR $\delta$ u $2.4,5.4,32.6,41.1,43.0,119.3,136.5,136.9,154.4 ; \mathrm{d} 13.6,21.7,26.9,52.2,52.5$, 55.4, 109.0, 118.8, 124.0, 135.9; IR (film, $\mathrm{cm}^{-1}$ ) 2942, 2861, 1575, 1458, 1100; HRMS calcd for $\mathrm{C}_{19} \mathrm{H}_{24} \mathrm{O}\left(\mathrm{M}^{+}\right)$268.1827, obsd 268.1824.

\section{(1R,3aR,9bR)-1-Cyclopropyl-6-methoxy-3a,8-dimethyl-2,3,3a,4,5,9b-hexahydro-1H- cyclopenta[ $\alpha]$ naphthalene-4,5-diol (25)}

The alkene 24 (200 mg, $0.745 \mathrm{mmol})$ was combined with $\mathrm{CCl}_{4}(4 \mathrm{~mL})$, water $(1 \mathrm{~mL})$, acetone $(8 \mathrm{~mL})$ and $t$ - $\mathrm{BuOH}(4 \mathrm{~mL})$ at rt. Potassium osmium (VI) oxide dihydrate $(14 \mathrm{mg}, 5 \% \mathrm{~mol})$ and $N$-methylmorpholine- $N$-Oxide $(0.41 \mathrm{~g}, 3.50 \mathrm{mmol})$ were added at once. ${ }^{3,18}$ The reaction mixture was stirred vigorously at $\mathrm{rt}$ for 2 days, then partitioned between MTBE and, sequentially, saturated aqueous $\mathrm{NH}_{4} \mathrm{Cl}$ and brine. The combined organic extract was dried $\left(\mathrm{Na}_{2} \mathrm{SO}_{4}\right)$ and concentrated in vacuo. The residue was chromatographed to afford the diol $\mathbf{2 5}$ as a mixture of diastereomers $(96 \mathrm{mg}, 0.317 \mathrm{mmol}, 43 \%$ yield, $60 \%$ yield based on starting material not recovered). Starting material was recovered $\left(55 \mathrm{mg}, 28 \%\right.$ yield, TLC $R_{f}(\mathrm{PE} / \mathrm{MTBE}$ $=6 / 4)=0.80)$. TLC $R_{f}(\mathrm{PE} / \mathrm{MTBE}=6 / 4)=0.29 ;{ }^{1} \mathrm{H} \mathrm{NMR} \delta-0.20-0.40(4 \mathrm{H}, \mathrm{m}), 0.54(1 \mathrm{H}, \mathrm{m})$, $1.17(1.5 \mathrm{H}, \mathrm{s}), 1.19(1.5 \mathrm{H}, \mathrm{s}), 1.25(3 \mathrm{H}, \mathrm{s}), 1.53(6 \mathrm{H}, \mathrm{m}), 1.88(1 \mathrm{H}, \mathrm{m}), 2.09(1 \mathrm{H}, \mathrm{m}), 2.18$ $(1 \mathrm{H}, \mathrm{s}), 2.32(3 \mathrm{H}, \mathrm{s}), 2.40(1 \mathrm{H}, \mathrm{m}), 2.63(1 \mathrm{H}, \mathrm{s}), 2.96(1 \mathrm{H}, \mathrm{d}, \mathrm{J}=8.0 \mathrm{~Hz}), 3.05(1 \mathrm{H}, \mathrm{d}, \mathrm{J}=$ 9.2Hz), $3.60(1 \mathrm{H}, \mathrm{d}, \mathrm{J}=4.0 \mathrm{~Hz}), 3.85(1.5 \mathrm{H}, \mathrm{s}), 3.87(1.5 \mathrm{H}, \mathrm{s}), 3.93(1 \mathrm{H}, \mathrm{m}), 5.06(1 \mathrm{H}, \mathrm{dd}$, $\mathrm{J}=4.4,8.0 \mathrm{~Hz}), 6.54(1 \mathrm{H}, \mathrm{s}), 6.68(0.5 \mathrm{H}, \mathrm{s}), 6.82(0.5 \mathrm{H}, \mathrm{s}) ;{ }^{13} \mathrm{C}$ NMR $\delta \mathrm{u} 2.99,3.76,5.67$, 6.09, 29.9, 31.3, 33.7, 36.9, 43.6, 44.4, 121.6, 122.0, 137.0, 137.1, 137.3, 138.0, 157.1, 157.3; d 12.2, 14.0, 21.2, 21.3, 28.8, 29.8, 49.2, 51.5, 53.1, 53.7, 54.8, 54.9, 65.0, 65.9, 71.4, 74.4, 107.8, 108.0, 123.7, 124.1; IR (film, cm ${ }^{-1}$ ) 2955, 1709, 1677, 1459, 1107; HRMS calcd for $\mathrm{C}_{19} \mathrm{H}_{26} \mathrm{O}_{3} \mathrm{Na}(\mathrm{M}+\mathrm{Na}) 325.1780$, obsd 325.1772 .

\section{(1R,3aR,9bR)-1-Isopropyl-6-methoxy-3a,8-dimethyl-2,3,3a,4,5,9b-hexahydro-1H-cyclopenta [a]naphthalen-4-ol (26)}

The diol 25 (36 mg, $0.12 \mathrm{mmol})$ and $\mathrm{PtO}_{2}(10 \mathrm{mg})$ were mixed in $\mathrm{AcOH}(2.5 \mathrm{~mL})$ at rt. $\mathrm{H}_{2}(1$ $\mathrm{atm})$ was bubbled through the mixture as it was maintained in an oil bath $\left(50{ }^{\circ} \mathrm{C}\right) .7 \mathrm{~b}, 19$ The reaction was monitored by TLC. When the reaction completed (about $1 \mathrm{~h}$ ), it was diluted with $20 \mathrm{~mL}$ of $\mathrm{Et}_{2} \mathrm{O}$ and passed through a pad of celite. The eluant was concentrated and chromatographed to afford the alcohol 26 as a colorless oil (23 $\mathrm{mg}, 0.079 \mathrm{mmol}, 67 \%$ yield). TLC $R_{f}(\mathrm{PE} / \mathrm{MTBE}=8 / 2)=0.25 ;{ }^{1} \mathrm{H}$ NMR $(360 \mathrm{MHz}) \delta 0.66(3 \mathrm{H}, \mathrm{d}, \mathrm{J}=6.5 \mathrm{~Hz}), 0.96(1 \mathrm{H}$, $\mathrm{m}), 1.09(3 \mathrm{H}, \mathrm{d}, \mathrm{J}=6.5 \mathrm{~Hz}), 1.13(1 \mathrm{H}, \mathrm{m}), 1.30(3 \mathrm{H}, \mathrm{s}), 1.48(1 \mathrm{H}, \mathrm{m}), 1.61(1 \mathrm{H}, \mathrm{m}), 1.86(1 \mathrm{H}$, $\mathrm{m}), 2.11(1 \mathrm{H}, \mathrm{m}), 2.30(3 \mathrm{H}, \mathrm{s}), 2.38(1 \mathrm{H}, \mathrm{m}), 3.01(2 \mathrm{H}, \mathrm{m}), 3.55(1 \mathrm{H}, \mathrm{dd}, \mathrm{J}=4.3,11.5 \mathrm{~Hz})$, $3.79(3 \mathrm{H}, \mathrm{s}), 6.51(1 \mathrm{H}, \mathrm{s}), 6.60(1 \mathrm{H}, \mathrm{s}) ;{ }^{13} \mathrm{C}$ NMR $(90 \mathrm{MHz}) \delta$ u $28.3,29.7,31.9,45.7,122.5$, 135.0, 138.2, 156.3; d 21.5, 21.7, 23.9, 26.4, 29.6, 53.3, 53.9, 55.3,75.7, 108.2, 122.7; IR (film, $\left.\mathrm{cm}^{-1}\right)$ 2924, 2855, 1458, 1264; HRMS calcd for $\mathrm{C}_{19} \mathrm{H}_{28} \mathrm{O}_{2}\left(\mathrm{M}^{+}\right)$288.2089, obsd 288.2088.

\section{(1R,3a $R, 9 \mathrm{~b} R)$-1-Isopropyl-6-methoxy-3a,8-dimethyl-1,2,3,3a-tetrahydro-9b $H$-cyclopenta[a] naphthalene-4,5-dione (27)}

The alcohol 26 (68 mg, $0.236 \mathrm{mmol}$ ), powdered 4A molecular sieve ( $0.65 \mathrm{~g}), \mathrm{N}$ methylmorpholine- $N$-Oxide $(272 \mathrm{mg}, 2.32 \mathrm{mmol}$ ) and TBAP (tetra- $n$-butylammonium perruthenate $)^{20}(10 \mathrm{mg})$ were mixed in DCM $(3 \mathrm{~mL})$ at $\mathrm{rt}$. The reaction was monitored by TLC. After about $3 \mathrm{~h}$, it was chromatographed directly to afford the known ${ }^{3}$ diketone $\mathbf{2 7}$ as a yellow oil (38 mg, $0.127 \mathrm{mmol}, 54 \%$ yield). TLC $R_{f}(\mathrm{PE} / \mathrm{MTBE}=8 / 2)=0.08 ;[\alpha]^{20} \mathrm{D}=-345.9^{\circ}$ (c $\left.0.74, \mathrm{CH}_{2} \mathrm{Cl}_{2}\right) ;{ }^{1} \mathrm{H}$ NMR $(360 \mathrm{MHz}) \delta 0.44(3 \mathrm{H}, \mathrm{d}, \mathrm{J}=6.5 \mathrm{~Hz}), 0.57(3 \mathrm{H}, \mathrm{d}, \mathrm{J}=6.5 \mathrm{~Hz})$, $1.20(1 \mathrm{H}, \mathrm{m}), 1.28(3 \mathrm{H}, \mathrm{s}), 1.55(2 \mathrm{H}, \mathrm{m}), 1.79(1 \mathrm{H}, \mathrm{m}), 2.25(1 \mathrm{H}, \mathrm{m}), 2.43(3 \mathrm{H}, \mathrm{s}), 2.50(1 \mathrm{H}$, $\mathrm{m}), 3.35(1 \mathrm{H}, \mathrm{d}, \mathrm{J}=9.7 \mathrm{~Hz}), 3.95(3 \mathrm{H}, \mathrm{s}), 6.71(1 \mathrm{H}, \mathrm{s}), 6.78(1 \mathrm{H}, \mathrm{s}) ;{ }^{13} \mathrm{C} \mathrm{NMR}(90 \mathrm{MHz}) \delta \mathrm{u}$ 
28.2, 35.6, 55.3, 120.8, 146.1, 147.4, 161.6, 181.0, 201.9; d 20.5, 22.6, 23.3, 24.3, 28.7, 52.0,

$56.2,56.2,111.1,124.5$.

(1R,3a $R, 9 \mathrm{~b} R$ )-6-Hydroxy-1-isopropyl-3a,8-dimethyl-1,2,3,3a-tetrahydro-9bH-cyclopenta[a] naphthalene-4,5-dione (28)

Following Clive's procedure ${ }^{3}$, the phenol $\mathbf{2 8}$ was isolated as a yellow solid (88\% yield). TLC $R_{f}(\mathrm{PE} / \mathrm{MTBE}=8 / 2)=0.46 ;[\alpha]^{20}{ }_{\mathrm{D}}=-225.8^{\circ}\left(\mathrm{c} 0.62, \mathrm{CH}_{2} \mathrm{Cl}_{2}\right) ;{ }^{1} \mathrm{H} \mathrm{NMR} \delta 0.43(3 \mathrm{H}, \mathrm{d}, \mathrm{J}=$ $6.8 \mathrm{~Hz}), 0.54(3 \mathrm{H}, \mathrm{d}, \mathrm{J}=6.8 \mathrm{~Hz}), 1.22(1 \mathrm{H}, \mathrm{m}), 1.30(3 \mathrm{H}, \mathrm{s}), 1.55(1 \mathrm{H}, \mathrm{m}), 1.67(1 \mathrm{H}, \mathrm{m}), 1.79$ $(1 \mathrm{H}, \mathrm{m}), 2.26(1 \mathrm{H}, \mathrm{m}), 2.39(3 \mathrm{H}, \mathrm{s}), 2.64(1 \mathrm{H}, \mathrm{m}), 3.40(1 \mathrm{H}, \mathrm{d}, \mathrm{J}=9.2 \mathrm{~Hz}), 6.70(1 \mathrm{H}, \mathrm{s}), 6.73$ $(1 \mathrm{H}, \mathrm{s}), 11.90(1 \mathrm{H}, \mathrm{s}) ;{ }^{13} \mathrm{C}$ NMR $\delta$ u $27.1,34.0,57.0,116.9,144.3,150.9,164.8,184.5,200.2$; d 20.0, 22.7, 23.3, 24.6, 28.3, 51.6, 56.7, 116.4, 123.5 .

\section{(-)-Hamigeran B (1)}

Following Trost's procedure ${ }^{4}, \mathbf{1}$ was isolated as a yellow solid ( $81 \%$ yield). The product was recrystallized from hexanes before it was characterized. m.p. $=155-160{ }^{\circ} \mathrm{C}\left(\right.$ lit. $1^{-4}$ m.p. 154 $\left.-167^{\circ} \mathrm{C}\right)$; TLC $R_{f}(\mathrm{PE} / \mathrm{MTBE} / \mathrm{MeOH}=90 / 10 / 1)=0.47 ;[\alpha]^{20} \mathrm{D}=-200^{\circ}\left(\mathrm{c} 0.14, \mathrm{CH}_{2} \mathrm{Cl}_{2}\right)$ (lit. $\left.1^{-4}[\alpha]_{\mathrm{D}}=-151.1^{\circ}--211^{\circ}\right) ;{ }^{1} \mathrm{H}$ NMR $\delta 0.45(3 \mathrm{H}, \mathrm{d}, \mathrm{J}=6.4 \mathrm{~Hz}), 0.54(3 \mathrm{H}, \mathrm{d}, \mathrm{J}=6.8 \mathrm{~Hz}), 1.20$ $(1 \mathrm{H}, \mathrm{m}), 1.30(3 \mathrm{H}, \mathrm{s}), 1.54(1 \mathrm{H}, \mathrm{m}), 1.68(1 \mathrm{H}, \mathrm{m}), 1.80(1 \mathrm{H}, \mathrm{m}), 2.30(1 \mathrm{H}, \mathrm{m}), 2.51(3 \mathrm{H}, \mathrm{s})$, $2.64(1 \mathrm{H}, \mathrm{m}), 3.38(1 \mathrm{H}, \mathrm{d}, \mathrm{J}=9.2 \mathrm{~Hz}), 6.83(1 \mathrm{H}, \mathrm{s}), 12.63(\mathrm{~s}, 1 \mathrm{H}) ;{ }^{13} \mathrm{C}$ NMR $\delta$ u $26.9,34.0$, $57.1,111.8,116.4,142.9,150.4,161.0,184.6,199.2$; d 19.9, 23.5, 24.5, 24.6, 28.3, 51.5, 56.4, 124.4 .

\section{Supplementary Material}

Refer to Web version on PubMed Central for supplementary material.

\section{Acknowledgment}

We thank John Dykins for high-resolution mass spectra under the financial support by NSF 0541775. This work was supported by the National Institutes of Health (GM 060287).

\section{REFERENCES}

(1). Wellington KD, Cambie RC, Rutledge PS, Bergquist PR. J. Nat. Prod 2000;63:79. [PubMed: 10650083]

(2). (a) Nicolaou KC, Gray D, Tae J. Angew Chem., Int. Ed 2001;40:3675. (b) Nicolaou KC, Gray D, Tae J. Angew Chem., Int. Ed 2001;40:3679. (c) Nicolaou KC, Gray DLF, Tae J. J. Am. Chem. Soc 2004;126:613. [PubMed: 14719961]

(3). (a) Clive DLJ, Wang J. Angew. Chem., Int. Ed 2003;42:3406. (b) Clive DLJ, Wang J. Tetrahedron Lett 2003;44:7731. (c) Clive DLJ, Wang J. J. Org. Chem 2004;69:2773. [PubMed: 15074927]

(4). (a) Trost BM, Pissot-Soldermann C, Chen I, Schroeder GM. J. Am. Chem. Soc 2004;126:4480. [PubMed: 15070341] (b) Trost BM, Pissot-Soldermann C, Chen I. Chem. Eur. J 2005;11:951.

(5). For a review of natural products synthesized from citronellal, see:Lenardao EJ, Botteselle GV, Azambuja F, Perin G, Jacob RG. Tetrahedron 2007;63:6671.

(6). Overman LE, Jacobsen EJ. J. Am. Chem. Soc 1982;104:7225.

(7). For the first reports of Rh-mediated intramolecular C-H insertion, see(a) Wenkert E, Davis LL, Mylari BL, Solomon MF, da Silva RR, Shulman S, Warnet RJ, Ceccherelli P, Curini M, Pellicciari R. J. Org. Chem 1982;47:3242. (b) Taber DF, Petty EH. J. Org. Chem 1982;47:4808.For a review of natural products synthesized by Rh-mediated intramolecular C-H insertion reaction, see: (c) Taber DF, Stiriba SE. Chem. Eur. J 1998;4:990.For a recent example, see (d) Taber DF, Frankowski KJ. J. Org. Chem 2005;70:6417. [PubMed: 16050704]

(8). Taber DF, Petty EH, Raman K. J. Am. Chem. Soc 1985;107:196.

(9). Mateos AF, Coca GP, Alonso JJP, González RR, Hernández CT. Synlett 1996:1134. 
(10). For the first report of intermolecular Rh-mediated C-H insertion using $\alpha$-aryl- $\alpha$-diazo esters, seeDavies HML, Hansen T. J. Am. Chem. Soc 1997;119:9075.For recent reviews, see (b) Davies HML, Beckwith REJ. Chem. Rev 2003;103:2861. [PubMed: 12914484] (c) Davies HML, Nikolai J. Org. Biomol. Chem 2005;3:4176. [PubMed: 16294245]

(11). Taber DF, Tian W. J. Org. Chem 2007;72:3207. [PubMed: 17385917]

(12). Anada M, Hashimoto S. Tetrahedron Lett 1998;39:79. $\mathrm{Rh}_{2}(\mathrm{Pttl})_{4}$ is dirhodium (II) tetrakis $[N$ phthaloyl-(S)-t-leucinate)

(13). Guggisberg Y, Faigl F, Schlosser M. J.Organomet. Chem 1991;415:1.

(14). Hunsen M. Tetrahedron Lett 2005;46:1651.

(15). Node M, Imazato H, Kurosaki R, Kawano Y, Inoue T, Nishide K, Fuji K. Heterocycles 1996;42:811.

(16). (a) Petasis NA, Bzowel EI. Tetrahedron Lett 1993;43:943. (b) Petasis NA, Bzowej EI. J. Am. Chem. Soc 1990;112:8392.

(17). (a) Nishimura S, Mochizuki F, Kobayakawa S. Bull. Chem. Soc. Jpn 1970;43:1919. (b) Nishimura S, Sakamoto H, Ozawa T. Chem. Lett 1973:855.

(18). VanRheenen V, Kelly RC, Cha DY. Tetrahedron Lett 1976:1973.

(19). Oppolzer W, Godel T. J. Am. Chem. Soc 1978;100:2583.

(20). Griffith WP, Ley SV, Whitcombe GP, White AD. J. Chem. Soc., Chem. Commun 1987:1625.

(21). ${ }^{13} \mathrm{C}$ multiplicities were determined with the aid of a JVERT pulse sequence, differentiating the signals for methyl and methine carbons as "d" and for methylene and quaternary carbons as " $u$ ". 
<smiles>Cc1cc2c(c(O)c1Br)C(=O)C(=O)C1(C)CC[C@@H](C(C)C)[C@H]21</smiles>

1 hamigeran $B$<smiles>[X]c1c(C)cc2c(c1O)C(=O)C(O)(C(C)=O)C(C)(C)C2C(C)C</smiles>

$3 \mathrm{X}=\mathrm{Br}$, hamigeran $\mathrm{A}$

$4 \mathrm{X}=\mathrm{H}$, debromohamigeran $\mathrm{A}$<smiles>CC(=O)O[C@H]1C(=O)C2=N[C@@H](C)Oc3c(Br)c(C)cc(c32)[C@H]2[C@H](C(C)C)CC[C@]12C</smiles>

6 hamigeran D<smiles>Cc1c(Br)c(O)c2c(c1Br)[C@H]1[C@@H](C(C)C)CC[C@@]1(C)C2=O</smiles>

2 4-bromohamigeran B<smiles>CC(=O)O[C@H]1C(=O)C(=O)c2c(cc(C)c(Br)c2O)[C@H]2[C@H](C(C)C)CC[C@@]12C</smiles>

5 hamigeran $\mathrm{C}$

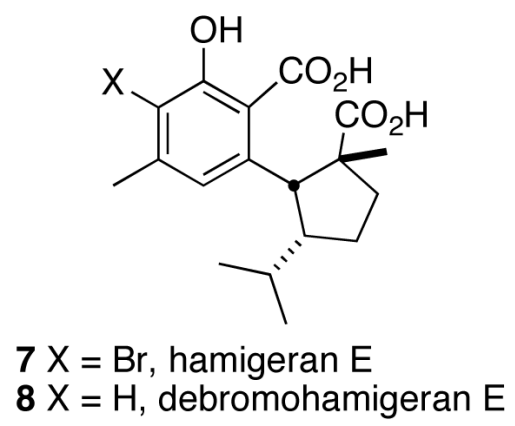

Figure 1.

Structures of Hamigerans. 
<smiles>COc1cc(COCCC(C)CCCC(=O)c2cc(C)cc(OC)c2)cc(C(=N)C(=O)CCC(C)CCCOc2ccccc2)c1</smiles>
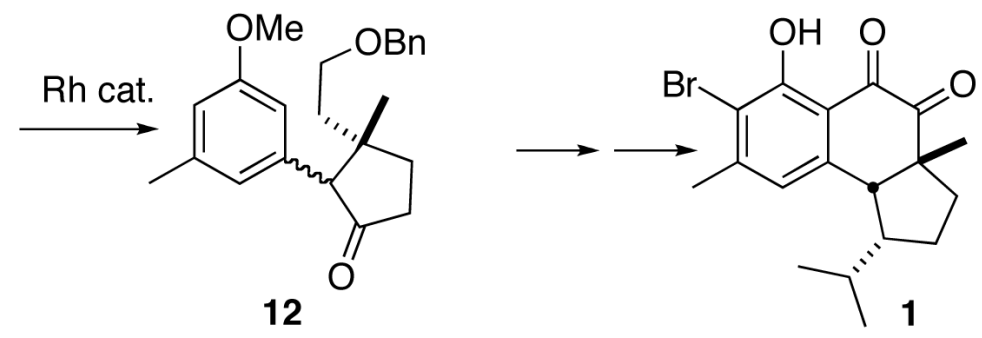<smiles>N=C(C(=O)CC1CCCCC1)c1ccccc1</smiles>

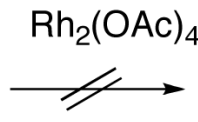

13

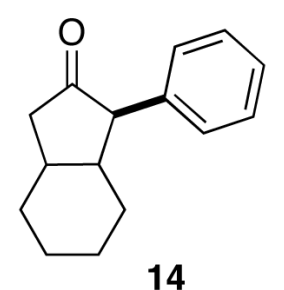

Scheme 1. 


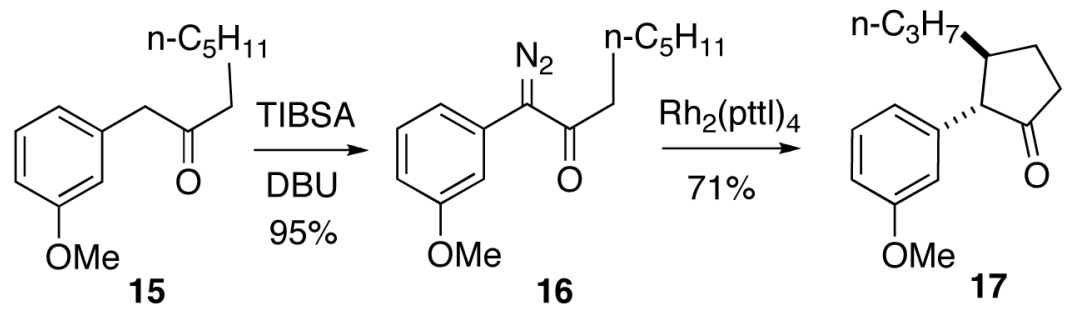<smiles>N=C(C(=O)CC1CCCCC1)c1ccccc1</smiles>

13

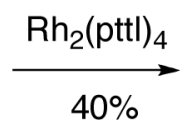

$40 \%$<smiles>O=C1CC2CCCCC2[C@H]1c1ccccc1</smiles>

14

Scheme 2. 


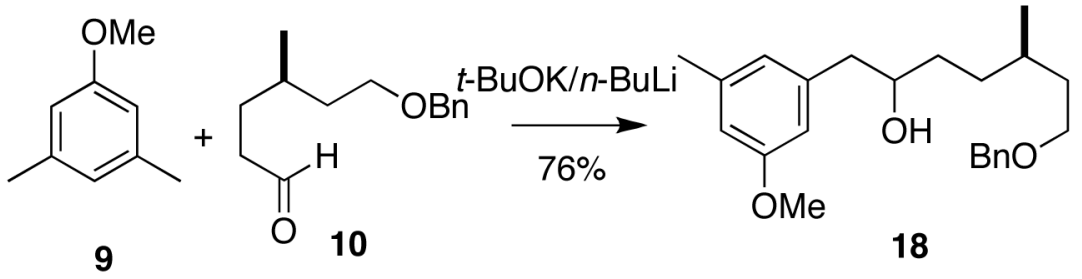

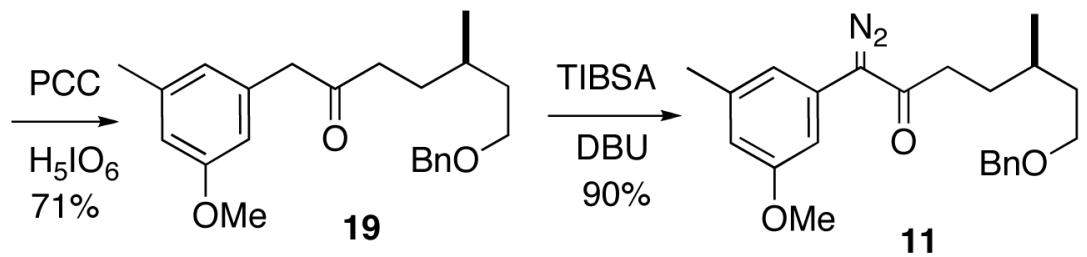<smiles>COc1cc(C)cc([C@H]2C(=O)CC[C@@]2(C)CCOc2ccccc2)c1</smiles><smiles>COc1cc(C)cc([C@H]2C(=O)CCC2(C)CCO)c1</smiles><smiles>CCCC1(C)CCC(=O)[C@@H]1c1cc(C)cc(OC)c1</smiles><smiles>CCO[SbH3]</smiles><smiles>COc1cc(C)cc2c1C=CC1(C)CCC(=O)C21</smiles>

Scheme 3. 
<smiles>COc1cc(C)cc2c1C=CC1(C)CCC(=O)C21</smiles>

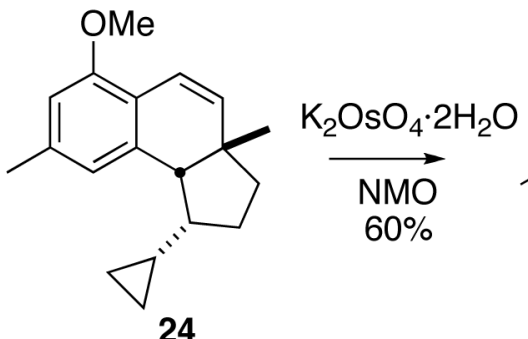<smiles>COc1cc(C)cc2c1C(O)C(O)C1(C)CC[C@H](C3CC3)[C@H]21</smiles><smiles>COc1cc(C)cc2c1CC(O)C1(C)CC[C@@H](C(C)C)[C@H]21</smiles><smiles>COc1cc(C)cc2c1C(=O)C(=O)C1(C)CC[C@@H](C(C)C)[C@H]21</smiles><smiles>Cc1cc(O)c2c(c1)[C@H]1[C@H](C(C)C)CC[C@]1(C)C(=O)C2=O</smiles>

28<smiles>Cc1cc2c(c(O)c1Br)C(=O)C(=O)C1(C)CC[C@H](C(C)C)[C@H]21</smiles>

(-)-Hamigeran B 1

Scheme 4. 